\title{
Imaging Magnetic Domains in Functional Nanoscale Heterostructures using Lorentz microscopy
}

\author{
C. Phatak ${ }^{1}$, F. Barrows ${ }^{1}$, V. Brajuskovic ${ }^{1}$, S. Bakaul ${ }^{1}$, O. Heinonen ${ }^{1}$, M. De Graef ${ }^{2}$, W. Jiang ${ }^{3}$, S. G. \\ E. te Velthuis ${ }^{1}$, A. Hoffmann ${ }^{1}$, A. Petford-Long ${ }^{1}$ \\ 1. Materials Science Division, Argonne National Laboratory, Lemont, IL, USA \\ 2. Department of Materials Science and Engineering, Carnegie Mellon University, Pittsburgh, PA \\ 15213, USA. \\ 3. Department of Physics, Tsinghua University, Beijing, P.R. China.
}

Lorentz transmission electron microscopy (LTEM) is ideally suited for quantitative analysis of magnetic domains at the nanometer length scale. The ability to study both the microstructure and mag-netic domain structure simultaneously in functional materials allows for direct understanding of the fundamental role of inhomogeneities in microstructure as well as the effect of shape and size of nanostructures on the magnetic domain behavior. The current state of art LTEM enabled using aberration correctors allows for imaging down to sub-nanometer scale in field-free conditions. With in-situ LTEM capabilities, the magnetic domain behavior can be studied as a function of external stimuli such as temperature or applied field, to gain a more detailed understanding of the fundamental physics. In this talk, we will present the application of aberration corrected LTEM to study the magnetic domain behavior in strongly interacting magnetic nanostructures as well as functional materials.

Topologically non-trivial spin textures such as skyrmions [1,2] present unique opportunities to explore exciting fundamental phenomena, such as the topological Hall effect, as well as novel applications, such as skyrmion-based spintronics. We will present application of LTEM to visualize and understand the magnetic spin textures in two types of systems that can host skyrmions: (1) $\mathrm{Ni}_{2} \mathrm{MnGa} \mathrm{Heusler}$ alloy system Figure 1(a) [3] and (2) $\left[(\mathrm{Pt}(1.5) / \mathrm{Co}(1) / \mathrm{W}(1)]_{8}\right.$ with broken inversion symmetry (Figure 1(b)). We will elucidate the nature of the skyrmions to be either Bloch-type or Néel-type as well as present in-situ studies of domain behavior in these systems to understand the stability of the skyrmions. We will also show that the chirality of both type of skyrmions can be unambiguously identified using LTEM.

Artificial spin ice lattices have recently emerged as a model system for studying magnetic frustration [4]. Most work to date has focused on the study of periodic artificial spin ice lattices. In our work, we have prepared artificial Permalloy spin ice lattices that have been patterned into quasicrystal P2 and P3 Penrose tilings (QC Lattices) that lack translational symmetry and are composed of vertices with a varying number of interacting elements as shown in Figure 2(a) and (b) respectively [5]. We will present a novel network topology analysis of these QC lattices based on graph theory methods. Using this method, we will show the emergent local magnetic ordering that occurs in such lattices during magnetization reversal. We will also discuss the thermalization behavior of these lattices [7].

References:

[1] A. Fert, N. Reyren, and V. Cros, Nat. Rev. Mater., 2, 17031 (2017).

[2] W. Jiang, et. al., Phys. Rep., 704, 1 (2017).

[3] C. Phatak, O. Heinonen, M. De Graef, and A. Petford-Long, Nano Lett., 16, 4141 (2016). 
[4] C. Nisoli, R. Moessner, and P. Schiffer, Rev. Mod. Phys., 85, 1473 (2013).

[5] V. S. Bhat, et. al., Phys. Rev. Lett, 111, 077201 (2013).

[6] V. Brajuskovic, F. Barrows, C. Phatak, A. K. Petford-Long, Sci. Rep,6, 34384 (2016).

[7] This work was supported by U.S. Department of Energy (DOE), Office of Science, Basic Energy Science, Materials Sciences and Engineering Division. Use of Center for Nanoscale Materials was supported by the U.S. Department of Energy, Office of Science, Office of Basic Energy Sciences, under contract no. DE-AC02-06CH11357.

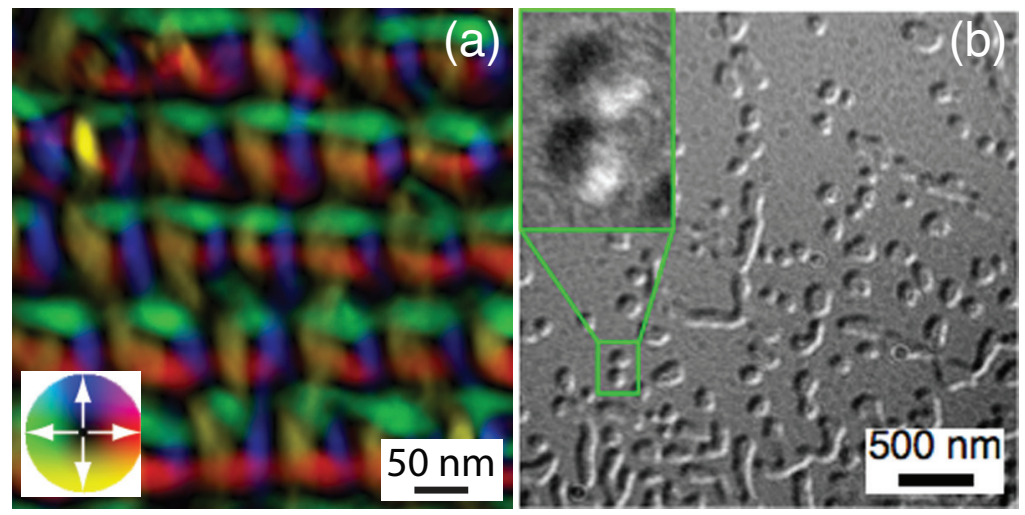

Figure 1. (a) Reconstructed color magnetic induction map of skyrmions formed in alternating twins of $\mathrm{Ni}_{2} \mathrm{MnGa}$, and (b) Under-focus LTEM image showing the formation of Néel-type skyrmions in $\left[(\operatorname{Pt}(1.5) / \mathrm{Co}(1) / \mathrm{W}(1)]_{8}\right.$ heterostructure.

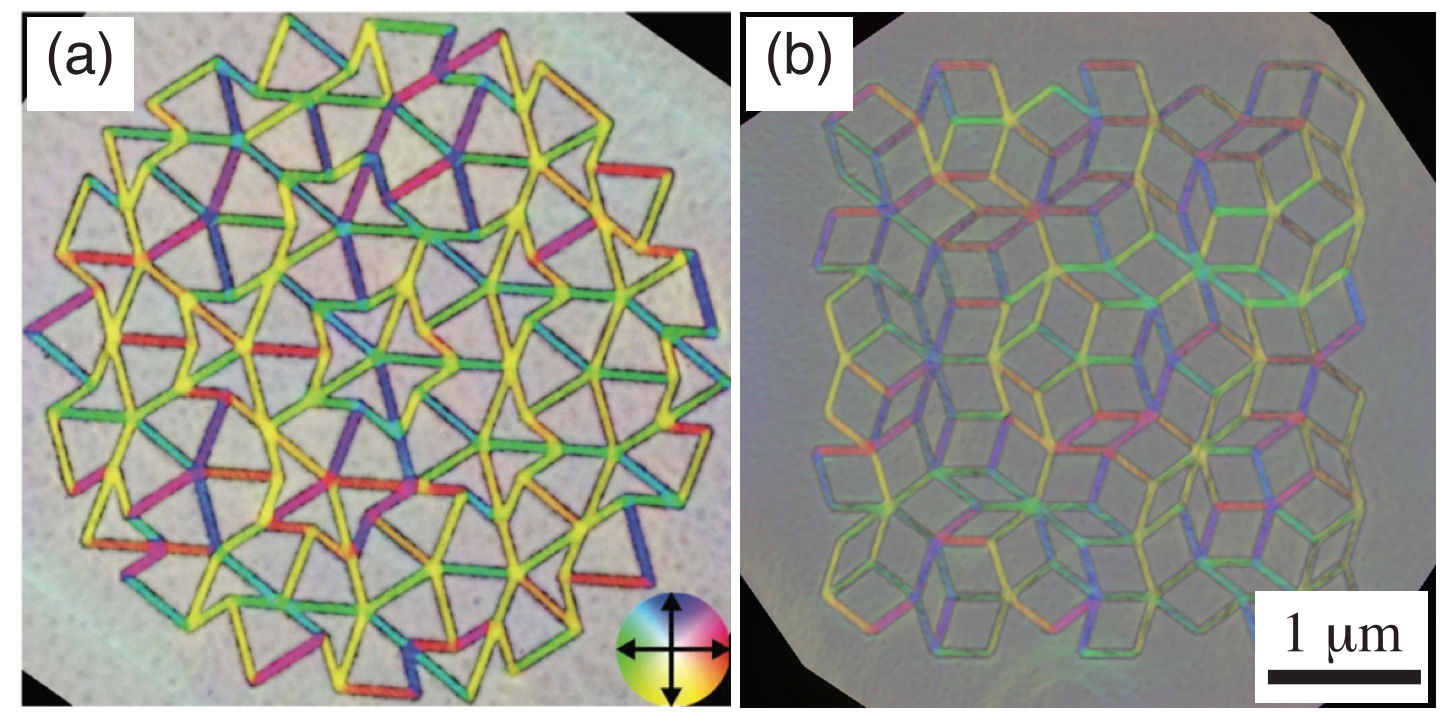

Figure 2. Reconstructed color magnetic induction maps of Penrose tiling after AC demagnetization protocol in (a) P2-type and (b) P3-type tiling. 